\title{
MEDICIÓN DEL NIVEL DE DESARROLLO DE LAS UNIVERSIDADES CHILENAS: UN ANÁLISIS CON MODELOS DE ECUACIONES ESTRUCTURALES
}

\author{
Cristian Mondaca ${ }^{1}$, \\ Jimmy Lopatinsky², \\ Alba Montecinos ${ }^{3}$, \\ Julio Rojas-Mora ${ }^{4}$
}

\section{RESUMEN}

En este trabajo se propone un indicador sintético que mide el grado de desarrollo de las universidades chilenas, valorando de forma cuantitativa las misiones (investigación y enseñanza) realizadas por las mismas. Se utiliza un modelo de ecuaciones estructurales resuelto a través de mínimos cuadrados parciales, analizando datos de 54 universidades de Chile, para el año 2017. Los resultados muestran que existen importantes diferencias entre universidades respecto del grado de desarrollo que presentan según la medición de la intensidad con que realizan las actividades de investigación y enseñanza. Se proponen medidas en el ámbito de las políticas públicas que apuntan a mejorar el sistema de educación universitaria y que ayudarían a reducir la brecha entre las universidades rezagadas y las que lideran el sistema.

Conceptos clave: indicador sintético, mínimos cuadrados parciales, modelo ecuaciones estructurales, ranking de universidades.

\section{MEASUREMENT OF THE DEVELOPMENT LEVEL OF CHILEAN UNIVERSITIES: AN ANALYSIS WITH STRUCTURAL EQUATION MODELS}

\section{ABSTRACT}

This paper proposes a synthetic indicator that measures the degree of development of Chilean universities, by quantitatively assessing each institution's missions (research and teaching). A model of structural equations solved through partial least squares is used, analyzing data from 54 universities in Chile, from the year, 2017. The results show that there are important differences among universities regarding the degree of development they present, according to the measure of the intensity with which they perform research and teaching activities. The article proposes public policy measures linked to improving the university education system that would help reduce the gap between the lowest performing universities and those that lead the system.

Key concepts: partial least squares, university rankings, synthetic indicator, structural equation modeling.

Universidad Austral de Chile, Valdivia, Chile. Contacto: cristianmondaca@uach.cl Universidad Austral de Chile, Valdivia, Chile. Contacto: jimmylopatinskyg@gmail.com Universidad Austral de Chile, Valdivia, Chile. Contacto: albamontecinos@uach.cl Universidad Católica de Temuco, Temuco, Chile. Contacto: jrojas@inf.uct.cl 


\section{Introducción}

Las universidades juegan un rol importante en la sociedad, ya que son consideradas un pilar fundamental en la formación de capital humano avanzado y principal fuente de generación de nuevo conocimiento en las diversas áreas de enseñanza; además, facilitan la transmisión de innovación a través de la investigación aplicada y la creación de patentes, todo esto para el beneficio de la sociedad (Scott, 2006).

Para cumplir este rol en la sociedad la universidad debe, por tanto, ser capaz de desarrollar las tradicionales misiones que la sociedad espera de ella, como son la investigación y la enseñanza, ya que estas contribuyen al desarrollo socioeconómico de las naciones (Gasset, 1946; Pacheco, 1997; Sánchez-Barrioluengo, 2014; Scott, 2006).

Sin embargo, se pueden observar diferencias entre universidades respecto de la intensidad con la cual realizan sus actividades vinculadas a dichas misiones y, por consiguiente, con la capacidad de cumplir el rol que la sociedad les asigna. Esto se puede deber a diversos factores, por ejemplo, diferencias en cuanto a sus años fundacionales o en las áreas del conocimiento que cultivan sus académicos y en las preferencias institucionales por desarrollar con mayor intensidad alguna de las misiones, entre otros aspectos. Debido a ello, realizar un análisis comparativo de universidades se vuelve una tarea muy compleja, dada la dificultad de valorar de forma adecuada el grado de desarrollo global o de las misiones que una universidad en particular ha alcanzado en un momento determinado.

En este contexto, los rankings se han convertido en una herramienta útil para valorar el grado de desarrollo que puede tener una universidad respecto de las demás, particularmente en un escenario donde existe un gran número de instituciones y diversidad de características (Reyes, 2016).

A pesar de ello, una dificultad presente en los rankings de las universidades es la manera en la que se agrega o sintetiza la información para facilitar la evaluación de su desempeño. Son conocidas las críticas en relación con la disparidad de criterios; la discrecionalidad de la selección de dimensiones, indicadores y ponderaciones; las formas 
de agregación que son utilizadas para construir un ranking; y la predominancia de indicadores bibliométricos y sus diferentes enfoques de valoración (Jajo \& Harrison, 2014; Luque-Martínez \& del BarrioGarcía, 2016; Reyes, 2016; Sanz-Casado, García-Zorita, SerranoLópez, Efraín-García y De Filippo, 2013), motivos por los cuales el resultado final de un ranking depende fuertemente de la selección realizada, donde las posiciones de las instituciones pueden cambiar significativamente, perjudicando o beneficiando a una universidad por sobre otra.

Una limitación que presentan los actuales rankings es la baja capacidad para mostrar las diferencias o brechas existentes entre instituciones y las dimensiones evaluadas, dado que se expresan en escalas ordinales, lo que dificulta determinar o dimensionar el esfuerzo que debe realizar una institución para mejorar su posición relativa. Este tipo de valoración puede ser relevante si se desea mejorar la situación de una universidad en particular, y dónde debe focalizar sus esfuerzos y recursos para mejorar su nivel de desarrollo.

Este estudio desea contribuir con una propuesta metodológica respecto de las dimensiones e indicadores que pueden ser utilizados para evaluar el grado de desarrollo que ha alcanzado una universidad y su posición relativa en relación con las demás, utilizando un modelo de ecuaciones estructurales resuelto a través de mínimos cuadrados parciales que, aplicado al caso de las universidades chilenas, permite generar un indicador sintético que mide el grado de desarrollo de una universidad y de sus misiones (investigación y enseñanza), y de cómo estas contribuyen a su posición final.

El objetivo principal de la investigación es proponer un nuevo indicador sintético que utiliza una metodología que soluciona la problemática habitualmente criticada en la construcción de rankings: la discrecionalidad existente en la selección de la ponderación o forma de agregación de los indicadores utilizados para la confección de un ranking en particular. Además, se propone un modelo que considera las principales actividades que realizan las universidades, lo que redunda en una evaluación más objetiva e integral de los planteles universitarios. 
El artículo se estructura de la siguiente forma. En primera instancia se presenta la introducción, para luego continuar con una revisión acerca de los rankings universitarios, una conceptualización en torno al rol de las universidades, una breve explicación de las bases teóricas que justifican el modelo teórico, y una pequeña presentación de los modelos de ecuaciones estructurales resueltos por mínimos cuadrados parciales. Se continúa con la descripción de la metodología y los datos utilizados, para luego exponer los resultados, la discusión y las principales conclusiones.

\section{Marco de referencia}

\subsection{Rankings universitarios}

Los rankings son uno de los instrumentos más ampliamente utilizados para evaluar el desempeño o calidad de las universidades, y es habitual su uso para comparar unas con otras en un país en particular, o bien, entre universidades a nivel internacional.

Según Usher y Savino (2018), los rankings de universidades son listas de instituciones clasificadas en forma comparativa de acuerdo con un conjunto común de indicadores en orden descendente, los que son presentados en forma de tabla de posiciones.

Los rankings se han transformado en una fuente de reputación indirecta, en la medida en que las universidades pueden posicionarse en los niveles superiores y diferenciarse del resto de ellas; y, por tanto, existe el incentivo para que estas inviertan recursos y tiempo para participar en los diferentes rankings existentes (Reyes, 2016).

Además, los rankings pueden incidir en la toma de decisiones de los grupos de interés en torno a las universidades, dado que pueden influir en su elección dependiendo de sus fines (Hazelkorn, 2011). Por ejemplo, los organismos públicos pueden seleccionar a las universidades mejor posicionadas para distribuir recursos públicos; así mismo, las instituciones de otros países pueden tener interés en relacionarse con las mejores universidades de un país determinado, con lo cual una entidad mejor posicionada en un ranking puede tener una probabilidad mayor de colaboración internacional. 
Los rankings también suelen ser utilizados para clasificar y agrupar universidades según su valor a nivel individual, institucional y nacional (Taylor \& Braddock, 2007). A nivel individual, la información es principalmente un recurso para futuros estudiantes (pregrado y posgrado), que proporciona comparaciones de desempeño institucional y que facilita la elección (Luque-Martínez \& DoñaToledo, 2013). A nivel institucional, los administradores pueden usarlos para juzgar las fortalezas y debilidades comparativas de su universidad, en tanto que a nivel nacional, los rankings pueden proporcionar información útil al gobierno, a otros líderes de la educación superior y tomadores de decisión, y también a ciudadanos comunes para evaluar el éxito de las políticas estatales o nacionales de educación superior que consideren disminuir las disparidades entre organismos de educación superior.

Sin embargo, es necesario mencionar que los rankings son posibles de construir en la medida en que se cuente con información relevante de las principales características que presentan las universidades $\mathrm{y}$, por lo tanto, se requiere de un sistema de indicadores que recoja periódicamente información de las instituciones de educación superior. Además, estos deben estar enfocados en evaluar los aspectos relevantes de la universidad, de manera que sean útiles para realizar comparaciones objetivas entre entidades (Martínez, Robles, Hernández, Zendejas y Pérez, 2007).

Históricamente, la clasificación de las universidades comienza con la propuesta del psicólogo James Mckeen Cattell en 1910, quien plantea su clasificación y evaluación en un ranking. La primera clasificación oficial se dio en Estados Unidos en el año de 1970 con la Classification Carnegie de instituciones superiores, creada por la Fundación Carnegie para el Avance de la Enseñanza Superior.

En los ochenta, los rankings se vuelven populares a nivel internacional, cuando surge el America's Best Colleges que fue presentado por la revista US News and World Report. Esta es una de las primeras guías con mayor precisión para comparar a las instituciones de educación superior de Estados Unidos (Quiles y de Gracia, 2015). 
En el año 2003, aparece el ranking de la Shanghai Jiao Tong University, que es uno de los más conocidos internacionalmente. Su objetivo principal es medir la productividad científica de las universidades, sin embargo, también incluye en sus análisis factores como el prestigio, la docencia y el tamaño de las instituciones. Según Pérez Esparrells y Gómez Sancho (2010), este ranking ordena a las 500 mejores universidades del mundo de acuerdo con cuatro criterios: calidad del alumnado, calidad del cuerpo docente, productividad científica, y ponderación por tamaño de características multidimensionales de la institución.

En la actualidad, ambos autores opinan que junto con el anterior los rankings de universidades más importantes son:

- Academic ranking of world universities (ARWU) y el World university rankings del Times Higher Education Supplement; elaborados por el Center for World-Class Universities y el Institute of Higher Education of Shanghai Jiao Tong University de China, respectivamente.

- El Performance ranking of scientific papers for world universities, desarrollado por el Higher Education and Accreditation Council of Taiwan.

- El Leiden world ranking, elaborado por el Centre for Science and Technology Studies de la Leiden University.

- El Scimago institutions ranking, desarrollado por el grupo Scimago en España.

Estos rankings normalmente se enfocan en evaluar la investigación por medio de la combinación de indicadores bibliométricos y los ponderan arbitrariamente (Pérez Esparrells y Gómez Sancho, 2010).

Para el caso de Chile, los rankings más conocidos son el Ranking de calidad de las universidades chilenas-RCUCh, realizado por el diario El Mercurio; y el Ranking de universidades realizado por la revista Qué Pasa, que construye un índice agregado multidimensional ponderado de las universidades, utilizando información cuantitativa de carácter anual. Existen también trabajos de investigación que han 
propuesto taxonomías de clasificación de universidades en Chile, las cuales han intentado determinar grupos afines, más que clasificar posiciones relativas entre las instituciones (Muñoz y Blanco, 2013).

\subsection{El sistema universitario de Chile}

El sistema universitario de Chile está formado por 61 instituciones que se dividen en dos grupos: las universidades tradicionales - pertenecientes al Consejo de Rectores de las Universidades Chilenas (CRUCH), y que está integrado por 18 universidades públicas del país y nueve universidades particulares-; y las universidades privadas, compuestas por las 34 instituciones restantes. Es un sistema de origen reciente, si se compara con otros países, dado que las primeras universidades del país datan de finales del siglo XVIII. Hasta el año 1980 solo existían ocho universidades, principalmente de origen público. Después de un cambio en la ley de educación superior en Chile, en 1981 se abrió la posibilidad para la creación de universidades privadas; y a raíz de aquello, el sistema ha crecido en forma importante durante los últimos 38 años.

Según datos del Consejo Nacional de Educación (CNED) para el año 2017, el sistema estaba formado por 722.741 estudiantes de pre y posgrado (659.577 estudiantes de pregrado y 63.164 estudiantes de posgrado). Ofrecía 10.685 programas únicos, que equivalen a 264 tipos de carreras profesionales diferentes en pregrado y 2.513 programas de estudio de posgrado. Un $85 \%$ de la matrícula total cursaba programas conducentes a título profesional con licenciatura, un $5,9 \%$ a título profesional sin licenciatura y $4,4 \%$ a técnico de nivel superior. En el caso de los posgrados un $67 \%$ conduce al grado de magíster y un $8,7 \%$ a grado de doctor.

\subsection{Un modelo para medir el grado de desarrollo de las universidades}

El rol de la universidad en la sociedad se puede conceptualizar en función de las misiones que tradicionalmente le han sido asignadas: la investigación y la enseñanza (Scott, 2006). Cada una de ellas puede ser considerada como un constructo que define y determina las acciones preferentes que desarrollan las universidades. 
La investigación se puede definir como el conjunto de actividades que realiza la universidad para crear y transmitir nuevo conocimiento, en donde la producción de literatura científica y el desarrollo de proyectos de investigación científica asumen un rol protagónico, y se asocian al concepto de producción/productividad científica. Paralelamente a la generación de nuevo conocimiento, están las acciones de transmisión de nuevo conocimiento e innovación. Estas actividades - que habitualmente se realizan a través de la generación de proyectos de investigación aplicada o la creación de nuevos métodos y formas de hacer, y que introducen innovación en los sistemas sociales y productivos-, pueden definirse como de transferencia e innovación. Estas últimas tienen un rol protagónico en el modelo de innovación en espiral que menciona las virtudes del trabajo colaborativo entre empresas, Estado y universidades, y que se formaliza en el modelo triple hélice propuesto por Etzkowitz en el año 1997 (Etzkowitz y Leydesdorff, 2000). Hoy en día, se discute acerca de esta emergente tercera misión de la universidad (transferencia e innovación), dadas las crecientes necesidades que tienen los territorios para mejorar su competitividad. En este sentido, producción científica y transferencia e innovación, permiten definir la actividad de investigación, en donde las universidades con un desarrollo superior realizarán con mayor intensidad estas acciones.

Por su parte, la enseñanza contempla todas las actividades de docencia que se realizan en la universidad para formar a los profesionales e investigadores que la sociedad requiere. Estas acciones son posibles gracias a las actividades de docencia de excelencia que los académicos desarrollan, y a los proyectos educativos que generan las universidades. Así mismo, son factibles en la medida en que existen recursos para financiar las actividades de docencia y espacios físicos dedicados a la enseñanza.

También se requiere de una demanda o flujo de estudiantes que deseen formarse en los diferentes programas formativos de pre y posgrado de la universidad, ya que ello hace posible el financiamiento de la universidad y, al mismo tiempo, da vida a las actividades que realiza la institución en cuanto a investigación y formación avanzada. En este sentido, se entiende que las universidades con una mejor 
docencia (excelencia en la enseñanza), mayor cantidad de recursos (físicos y monetarios), y con un mayor flujo de estudiantes, estarán más desarrolladas.

Los conceptos mencionados anteriormente deben estar asociados con indicadores que reflejen en forma adecuada las misiones y actividades que desarrollan las universidades. La producción científica de estas instituciones puede ser medida utilizando los resultados de la investigación, esto es, los proyectos de investigación realizados y la cantidad de artículos científicos publicados (LuqueMartínez \& del Barrio-García, 2016; Martínez Rizo, 2011).

En el caso de las actividades de transferencia e innovación estas pueden medirse utilizando la cantidad de proyectos de investigación aplicada y los recursos captados por asesorías y servicios; además de la cantidad de patentes generadas por las universidades (Luque-Martínez $\&$ del Barrio-García, 2016).

Por su parte, la docencia de excelencia puede ser medida utilizando los indicadores que consideran la cantidad de académicos y su grado de perfeccionamiento. Ahora bien, puesto que la sola cantidad de académicos no da cuenta del grado de excelencia de la enseñanza impartida, se puede considerar la cantidad de académicos con formación de posgrado (magíster y doctorado), así como también los años de acreditación que han alcanzado las universidades y el número de áreas acreditadas.

En cuanto a los recursos para la enseñanza, estos pueden medirse indirectamente a partir de la infraestructura disponible para los estudiantes y académicos (Hernández, Leyva, Márquez y Cerda, 2014) y de los recursos financieros para el funcionamiento administrativo de la universidad y el pago de los salarios de sus académicos.

Por último, los procesos formativos pueden medirse indirectamente en función de la demanda de estudiantes para el ingreso a los programas de pre y posgrado de la universidad, además de la cantidad de titulados y graduados; es decir, estudiantes que han 
terminado el ciclo formativo y que son evidencia del funcionamiento de los procesos allí involucrados (Martínez Rizo, 2011).

En la Figura 1 se puede ver el modelo estructural propuesto que se inspira en el trabajo de Luque-Martínez y del Barrio-García (2016). En este caso, se presenta un modelo de ecuaciones estructurales en donde las variables latentes se manifiestan en un modelo tipo reflectivo-formativo (Becker, Klein \& Wetzels, 2012; Hair, Hult, Ringle \& Sarstedt, 2017), donde las variables latentes (en adelante VL), de primer orden son de tipo reflectivo y sus indicadores recogen las distintas formas en que se manifiestan, que en este caso son:

- la producción científica,

- la transferencia e innovación,

- la docencia de excelencia,

- los recursos y espacios de enseñanza, y

- los procesos formativos.

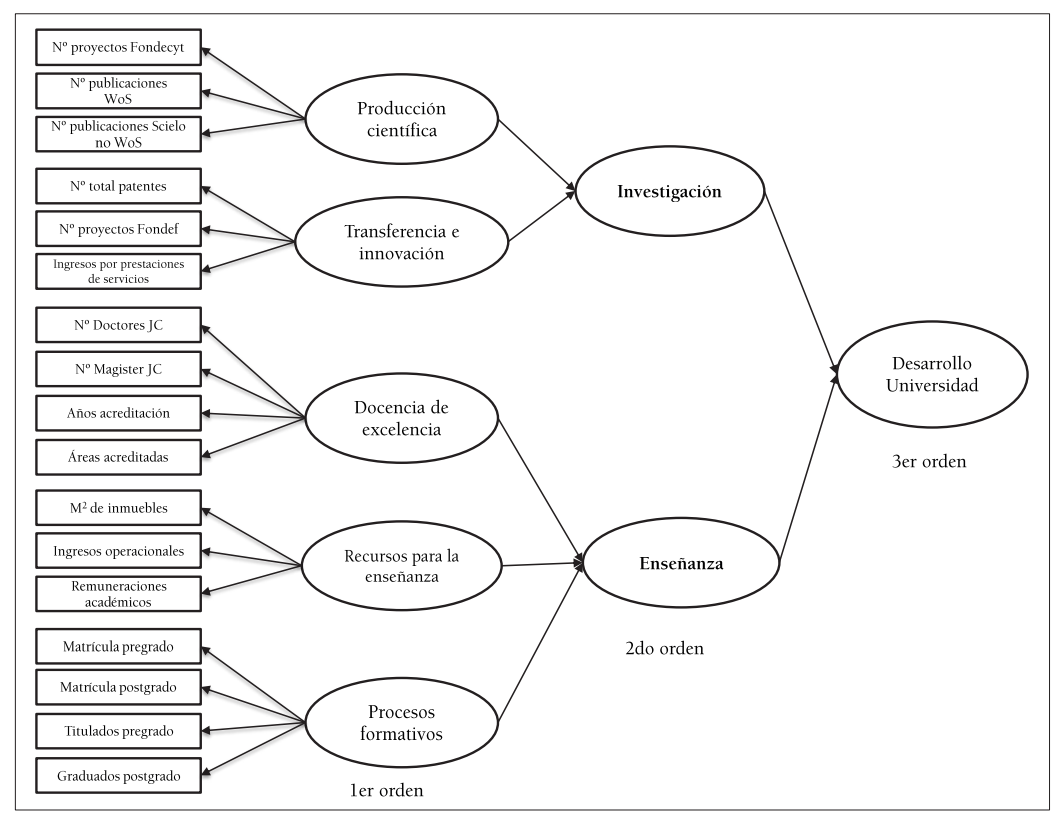

Figura 1. Modelo e indicadores propuestos.

Fuente: Elaboración propia. 
Las VL son medidas por indicadores reflectivos (o variables observadas) que tratan de recoger las distintas formas en las cuales se manifiestan, y en general se encuentran altamente correlacionadas en cada uno de los constructos. Las VL de segundo y tercer orden del tipo formativo son luego sintetizadas a partir de los constructos anteriores, por ejemplo, la actividad de investigación que realizan las universidades se debe a la contribución de la productividad científica y de la transferencia e innovación (Luque-Martínez \& del Barrio-García, 2016).

En el caso de la actividad de enseñanza, esta se debe a las actividades de docencia de excelencia, los recursos financieros y espacios físicos disponibles para la enseñanza, y remuneración de la docencia; y los procesos formativos que se desarrollan en la universidad.

La VL formativa de tercer orden denominada "desarrollo universidad", refleja las contribuciones de las actividades de investigación y de enseñanza, midiendo el grado de desarrollo que ha alcanzado una universidad respecto de las demás, considerando el aporte individual de cada una de las misiones.

\subsection{Los modelos estructurales resueltos con mínimos cuadrados parciales}

A modo resumido, los modelos estructurales resueltos con mínimos cuadrados parciales, en adelante, PLS-SEM (por su sigla en inglés Partial Least Squares Structural Equations Modeling) representan un conjunto de métodos estadísticos muy recientes que son utilizados principalmente para desarrollar teorías en la investigación exploratoria. Los PLS-SEM se enfocan en estimar una red teórica causal de relaciones que une conceptos complejos latentes, cada uno medido por medio de una serie de indicadores observables. Es una alternativa a la correlación canónica o el modelado de ecuaciones estructurales basado en la covarianza, debido a que el método busca maximizar la varianza explicada. Además, presenta las ventajas de manejar la multicolinealidad entre las variables independientes y entre las variables dependientes; robustez ante el ruido de datos y la falta de 
datos; y la posibilidad de trabajar con tamaños de muestra pequeños (Hair et al., 2017; Vinzi, Chin, Henseler \& Wang, 2010).

El PLS-SEM puede implementarse como un modelo de regresión, prediciendo una o más variables dependientes de un conjunto de una o más variables independientes; o bien, puede implementarse como un modelo de ruta, manejando trayectorias causales que relacionan los predictores, así como las rutas que relacionan los predictores con la(s) variable(s) de respuesta o VL. Además, no requiere suposiciones mínimas hechas para las escalas de medición, especificación de modelos de medición (indicadores reflexivos o formativos) y distribución de datos (Del Barrio-García y Luque Martínez, 2012).

Los modelos PLS-SEM se han usado recientemente en las áreas de las ciencias sociales, y en economía, particularmente en negocios y marketing (Hair et al., 2017; Sarstedt, Ringle \& Hair, 2014). En el ámbito de las universidades, se ha propuesto su uso para medir el desempeño de las mismas, por ejemplo, Jajo y Harrison (2014) presentaron una alternativa a los rankings de nivel internacional, y en el caso de Luque-Martínez y del Barrio-García (2016) los utilizaron para generar un ranking de la actividad investigadora de las universidades en España.

\section{Metodología y datos}

Para la construcción de un indicador sintético se han utilizado los datos del CNED, que pone a disposición una base de datos pública con diversa información de las instituciones de educación superior en Chile $^{5}$ e información relacionada con los alumnos que han ingresado cada año a las instituciones. Además, se ha complementado con información estadística del Consejo Nacional de Ciencia y Tecnología de Chile (Conicyt) ${ }^{6}$, respecto de información de patentes y proyectos de investigación y de investigación aplicada.

Mayores informaciones en cned.cl

Mayores informaciones en conicyt.cl 
En este estudio se ha reunido información de 54 universidades chilenas que representan aproximadamente el 88,5\% del total de universidades públicas y privadas existentes en el país para el año 2017. Una descripción detallada de los indicadores usados en el modelo se puede ver en el Anexo.

\subsection{El método de estimación}

El modelo aquí propuesto puede ser expresado como dos modelos lineales, uno interno y otro externo. El modelo interno es el principal, que describe la relación entre las VL, según la ecuación (1) (Jajo \& Harrison, 2014; Vinzi et al., 2010):

$$
\left.V L_{j}=\beta_{0}+\sum_{j} \sum_{i} \beta_{j i} V L_{i}+e_{i} 1\right)
$$

Tal que $\quad E\left(V L_{j} / V L_{i}\right)=\beta_{0 i}+\sum_{j} \sum_{i} \beta_{j i} V L_{i} \mathrm{y} \operatorname{cov}\left(V L_{j}, e_{i}\right)=0$.

Por otra parte, el modelo externo (a veces denominado modelo de medición), es la parte que tiene que ver con las relaciones entre una VL y su bloque de variables manifiestas (ecuación 2).

$$
X_{j k}=\lambda_{0 j k}+\lambda_{j k} V L_{j}+e_{j k}
$$

Tal que $\quad E\left(X_{j k} / V L_{j}\right)=\lambda_{0 j k}+\lambda_{j k} V L_{j}$

Los $V L_{j}$ estimados por PLS-SEM representarán el grado de desarrollo de los constructos propuestos. Las puntuaciones de las variables latentes se calculan como sumas ponderadas de sus indicadores (ecuación 3):

$$
\left.Y_{j}=\widehat{V L}_{j} \sum_{k} w_{j k} X_{k} 3\right)
$$

Los pesos externos $w_{j k}$ se estiman iterativamente como $\hat{w}_{j k}=\left(Y_{j} Y_{j}\right)^{-1} Y_{j} X_{j k}$, mientras que los coeficientes conectores estructurales (path, en inglés) $\hat{\beta}_{j i}$ son estimados de un modelo de regresión múltiple $Y_{j}=\sum_{i \rightarrow j} \hat{\beta}_{j i} Y_{i}$ usando el método de mínimos cuadrados $\hat{\beta}_{j i}=\left(Y_{i} Y_{i}\right)^{-1} Y_{i} Y_{j}$

La estimación de las cargas (loading, en inglés) $\hat{\lambda}_{j k}=\rho\left(X_{j k}, Y_{j}\right)$ se define como la correlación entre las VL y los indicadores. Dado que PLS-PM no depende de ningún supuesto de distribución, los niveles de significación de las estimaciones de los parámetros (según 
la teoría normal) son estimados utilizado la técnica de bootstrapping para obtener información acerca de la variabilidad de las estimaciones de los parámetros y, por lo tanto, validar el modelo.

El modelo propuesto ha sido estimado usando el software SmartPLS 3.0 (Ringle, Wende \& Becker, 2015), y para la estimación de la confiabilidad de sus parámetros se ha utilizado un bootstrapping de 5.000 muestras.

\section{Análisis de resultados ${ }^{7}$}

En la Tabla 1 se pueden ver los resultados del modelo estructural, en donde se presentan las cargas para cada uno de los indicadores y su significancia. Las cargas externas (outer loading) representan la contribución absoluta de un indicador en la definición de la VL, y se busca que los coeficientes de las cargas superen el valor 0,70; así como también, que todas las cargas sean significativas, es decir, con valores $t$-value $>1.96$ y valores $p<0.05$ (Hair et al., 2017).

7 En la Tabla 6 del Anexo se puede ver un resumen estadístico descriptivo de todos los indicadores utilizados en el modelo. 
298 MEDICIÓN DEL NIVEL DE DESARROLLO DE LAS UNIVERSIDADES CHILENAS: UN ANÁLISIS CON MODELOS DE ECUACIONES ESTRUCTURALES - C. Mondaca, J. Lopatinsky, A. Montecinos, J. Rojas-Mora

Tabla 1

Cargas estimadas del modelo y su significancia*

\begin{tabular}{llllll}
\hline Variable latente & \multicolumn{1}{c}{ Indicador } & Cargas & $\begin{array}{l}\text { Desviación } \\
\text { estándar }\end{array}$ & t-valor & p-valor \\
\hline $\begin{array}{lllll}\text { Producción } \\
\text { científica }\end{array}$ & $\mathrm{N}^{\circ}$ proyectos Fondecyt & 0,994 & 0,011 & 93,045 & $<0,000$ \\
\cline { 2 - 6 } & $\mathrm{N}^{\circ}$ publicaciones WoS & 0,992 & 0,011 & 89,756 & $<0,000$ \\
\cline { 2 - 6 } & $\begin{array}{l}\mathrm{N}^{\circ} \text { publicaciones SciELO } \\
\text { no ISI }\end{array}$ & 0,985 & 0,031 & 31,967 & $<0,000$ \\
& & & & &
\end{tabular}

\begin{tabular}{llllll}
\hline Transferencia & $\mathrm{N}^{\circ}$ total de patentes & 0,879 & 0,044 & 20,010 & $<0,000$ \\
\cline { 2 - 6 } $\begin{array}{l}\text { e investigación } \\
\text { aplicada }\end{array}$ & \begin{tabular}{l}
$\mathrm{N}^{\circ}$ proyectos Fondef \\
\cline { 2 - 6 }
\end{tabular} & 0,957 & 0,012 & 81,180 & $<0,000$ \\
\hline $\begin{array}{l}\text { Ingresos por prestación } \\
\text { servios }\end{array}$ & 0,640 & 0,235 & 2,727 & $<0,006$
\end{tabular}

\begin{tabular}{llcccc}
\hline $\begin{array}{l}\text { Docencia de } \\
\text { excelencia }\end{array}$ & $\mathrm{N}^{\mathrm{o} \text { doctores JC }}$ & 0,849 & 0,021 & 39,703 & $<0,000$ \\
\cline { 2 - 6 } & $\mathrm{N}^{\circ}$ magíster JC & 0,566 & 0,091 & 6,211 & $<0,000$ \\
\cline { 2 - 6 } & Años de acreditación & 0,913 & 0,025 & 36,051 & $<0,000$ \\
\cline { 2 - 6 } & $\mathrm{N}^{\circ}$ de áreas acreditadas & 0,886 & 0,021 & 41,886 & $<0,000$ \\
\hline $\begin{array}{l}\text { Recursos y } \\
\text { espacio para la } \\
\text { enseñanza }\end{array}$ & $\mathrm{M}^{2}$ de inmuebles & 0,847 & 0,104 & 8,112 & $<0,000$ \\
\cline { 2 - 6 } & Ingresos operacionales & 0,869 & 0,054 & 15,953 & $<0,000$ \\
\cline { 2 - 6 } & $\begin{array}{l}\text { Remuneraciones de } \\
\text { académicos }\end{array}$ & 0,849 & 0,053 & 16,041 & $<0,000$ \\
\hline $\begin{array}{l}\text { Procesos } \\
\text { formativos }\end{array}$ & Matrícula pregrado & 0,881 & 0,052 & 17,092 & $<0,000$ \\
\cline { 2 - 6 } & Matrícula posgrado & 0,900 & 0,028 & 32,276 & $<0,000$ \\
\cline { 2 - 6 } & Titulados pregrado & 0,820 & 0,071 & 11,600 & $<0,000$ \\
\cline { 2 - 6 } & Graduados de posgrado & 0,887 & 0,040 & 21,983 & $<0,000$ \\
\hline
\end{tabular}

* A través de la técnica de bootstrapping se analiza la robustez de las cargas de los indicadores y si las relaciones entre variables son significativas. Bootstrapping de 5.000 submuestras.

Fuente: Elaboración propia.

Se puede apreciar en la tabla anterior que casi todos los indicadores presentan valores mayores a 0,7 ; las excepciones son "Ingresos por prestación de servicios" y "No de magíster a jornada completa", lo que significa que contribuyen en menor medida a la explicación de la varianza de la VL. Sin embargo, no se pueden eliminar, dado que son significativos y, por tanto, solo se puede concluir que la contribución de estos indicadores a la varianza es menor, lo que se puede deber a que existen universidades que no presentan una actividad de transferencia importante y que capturan recursos de asesorías. En el caso del indicador " $\mathrm{N}^{\circ}$ de académicos con grado de magíster a jornada completa", la variabilidad entre 
universidades puede ser menor, es decir, probablemente no existen grandes diferencias entre las universidades de alto estándar que presentan un número mayor de doctores que de académicos con grado de magíster a jornada completa, versus las universidades con menores niveles de calidad, y que cuentan con un número mayor de académicos que solo tienen una licenciatura y un número menor de académicos con grado de magíster.

En la Tabla 2 se resume el análisis de la validez y fiabilidad de los constructos o VL medida a través de la fiabilidad compuesta CR (composite reliability, en inglés) y el promedio de la varianza extraída AVE (en inglés average variance extracted), las cuales presentan valores sobre sus límites recomendados $C R>0,7$ y AVE $>0,50$ respectivamente (Luque-Martínez \& del Barrio-García, 2016). Además, se complementa esta información con otros criterios habitualmente utilizados $\left(\alpha_{\text {Cronbach }}, \rho_{\mathrm{A}}\right)$.

Tabla 2

Análisis de la validez y fiabilidad de los constructos

\begin{tabular}{lcccc}
\hline Constructos & $\begin{array}{c}\alpha \text { de } \\
\text { Cronbach }\end{array}$ & $\rho_{\mathrm{A}}$ & $\begin{array}{c}\text { Varianza } \\
\text { extraída (AVE) }\end{array}$ & $\begin{array}{c}\text { Fiabilidad } \\
\text { compuesta (CR) }\end{array}$ \\
\hline Productividad científica & 0,990 & 0,990 & 0,994 & 0,981 \\
Transferencia y ciencia aplicada & 0,770 & 0,833 & 0,872 & 0,699 \\
Docencia de excelencia & 0,820 & 0,850 & 0,885 & 0,665 \\
Recursos y espacios para la & 0,817 & 0,823 & 0,891 & 0,731 \\
enseñanza & 0,896 & 0,904 & 0,927 & 0,761 \\
Procesos formativos & 0,938 & 0,960 & 0,954 & 0,779 \\
Investigación & 0,909 & 0,923 & 0,925 & 0,534 \\
Enseñanza & 0,948 & 0,961 & 0,955 & 0,564 \\
\hline Desarrollo universidad & & & & \\
\hline
\end{tabular}

Fuente: Elaboración propia.

En este caso, se puede ver que todas las variables latentes presentan valores superiores a los valores mínimos recomendados, y se pueden considerar constructos válidos y fiables para medir el grado de desarrollo de las actividades de investigación y enseñanza, y nivel de desarrollo global que tienen las universidades.

Para evaluar el modelo en su conjunto, se analizó la validez discriminante de los constructos de primer orden mediante el criterio 
de Fornell y Larcker (1981), valores que se pueden ver en la Tabla 3. Para ello, se usa la varianza extraída media (AVE, por sus siglas en inglés), la cual acepta un constructo como válido si el AVE resulta mayor a 0,5 ; en caso contrario, la varianza capturada por el constructo evaluado, y la validez de los respectivos indicadores individuales es cuestionable (Fornell \& Larcker ,1981).

Tabla 3

Validez discriminante (criterio de Fornell \& Larcker, 1981)

\begin{tabular}{lccccc}
\hline & a) & b) & c) & d) & e) \\
\hline a) Docencia excelencia & 0,815 & & & & \\
b) Procesos formativos & 0,767 & 0,872 & & & \\
c) Infraestructura y espacio para la enseñanza & 0,675 & 0,353 & 0,855 & & \\
d) Transferencia e investigación aplicada & 0,660 & 0,589 & 0,629 & 0,836 & \\
e) Producción científica & 0,754 & 0,725 & 0,635 & 0,854 & 0,990 \\
\hline
\end{tabular}

Fuente: Elaboración propia.

En este caso, todos los constructos superan el valor 0,5 , por lo cual efectivamente existe una relación entre las variables observadas y las latentes para medir el mismo fenómeno.

En la Tabla 4 se presentan los coeficientes estimados del modelo estructural y su nivel de significación a través del uso del bootstrapping. En principio, se asume una relación directa positiva entre los constructos, es decir, una mayor producción científica y una mayor transferencia e innovación inciden en una mayor actividad de investigación. Por otra parte, una mayor docencia de excelencia, más recursos físicos y financieros destinados a la enseñanza, y un flujo mayor de alumnos vinculados con los procesos formativos que realizan las universidades están asociados a una mayor actividad de enseñanza. 
Tabla 4

Significancia* de las variables latentes

\begin{tabular}{lcccc}
\hline Constructos & $\begin{array}{c}\text { Coeficiente } \\
\text { (Path } \\
\text { coefficients) }\end{array}$ & $\begin{array}{c}\text { Desviación } \\
\text { estándar }\end{array}$ & t-valor & p-valor \\
\hline $\begin{array}{l}\text { Investigación } \rightarrow \text { Desarrollo universidad } \\
\text { Enseñanza } \rightarrow \text { Desarrollo universidad }\end{array}$ & 0,470 & 0,029 & 15,971 & $<0,000$ \\
\hline Producción científica $\rightarrow$ Investigación & 0,578 & 0,027 & 21,750 & $<0,000$ \\
Transferencia e innovación $\rightarrow$ Investigación & 0,414 & 0,026 & 15,932 & $<0,000$ \\
\hline Docencia de excelencia $\rightarrow$ Enseñanza & 0,418 & 0,050 & 8,381 & $<0,000$ \\
$\begin{array}{l}\text { Recursos físicos y financieros para la } \\
\text { enseñanza } \rightarrow \text { Enseñanza }\end{array}$ & 0,284 & 0,043 & 6,689 & $<0,000$ \\
Procesos formativos $\rightarrow$ Enseñanza & 0,452 & 0,037 & 12,092 & $<0,000$ \\
\hline
\end{tabular}

Fuente: Elaboración propia.

Sin embargo, al considerar el tamaño de los conectores del modelo (path), se puede apreciar que los constructos no inciden de la misma forma en el grado de desarrollo que se puede observar en las universidades. Por ejemplo, en el caso de la actividad de investigación, la producción científica tiene un peso relativo mayor $(0,623)$, y en menor medida la transferencia e innovación $(0,414)$; este desequilibrio se debe a que las universidades están más orientadas a desarrollar productividad científica que a realizar actividades de transferencia e innovación. Esto se puede deber a que en el caso de Chile la productividad científica está asociada a la captación de recursos públicos hacia las universidades, con lo cual existe un importante incentivo para desarrollar productividad científica.

En el caso de la enseñanza, los constructos "procesos formativos" $(0,452)$ y "docencia de excelencia" $(0,418)$ tienen un peso mayor que los "recursos físicos y financieros para la enseñanza" $(0,284)$. Esto implica que las universidades están más orientadas a realizar procesos formativos y, en menor medida, a la docencia de excelencia, además de asociar recursos físicos y financieros en el desarrollo de sus actividades de enseñanza.

$\mathrm{Al}$ analizar las VL de segundo y tercer orden, se puede apreciar que la "actividad de enseñanza" $(0,578)$ tiene un peso relativo mayor respecto de la "actividad de investigación" $(0,470)$ en el nivel de desarrollo que muestran las universidades chilenas; por lo tanto, se 

ANÁLISIS CON MODELOS DE ECUACIONES ESTRUCTURALES - C. Mondaca, J. Lopatinsky, A. Montecinos, J. Rojas-Mora

evidencia que el sistema universitario para el año 2017 tiene una orientación mayor hacia la enseñanza que hacia la investigación. Esto puede deberse a que en el sistema universitario chileno la educación está privatizada, y a pesar de que existen universidades públicas, el sistema de financiamiento es vía voucher (vía asistencia) para los estudiantes más vulnerables y de autofinanciamiento para el resto, con lo que existe un incentivo para la captación de estudiantes y del desarrollo de la educación universitaria como un bien de consumo.

En cuanto a los valores individuales de las VL obtenidas para cada una de las universidades, estas se pueden observar en detalle en Tabla 6 del Anexo. Por su parte, en la Figura 2 se puede ver la relación entre la primera y segunda misión (investigación y enseñanza, respectivamente) alcanzada para cada una de las universidades chilenas.

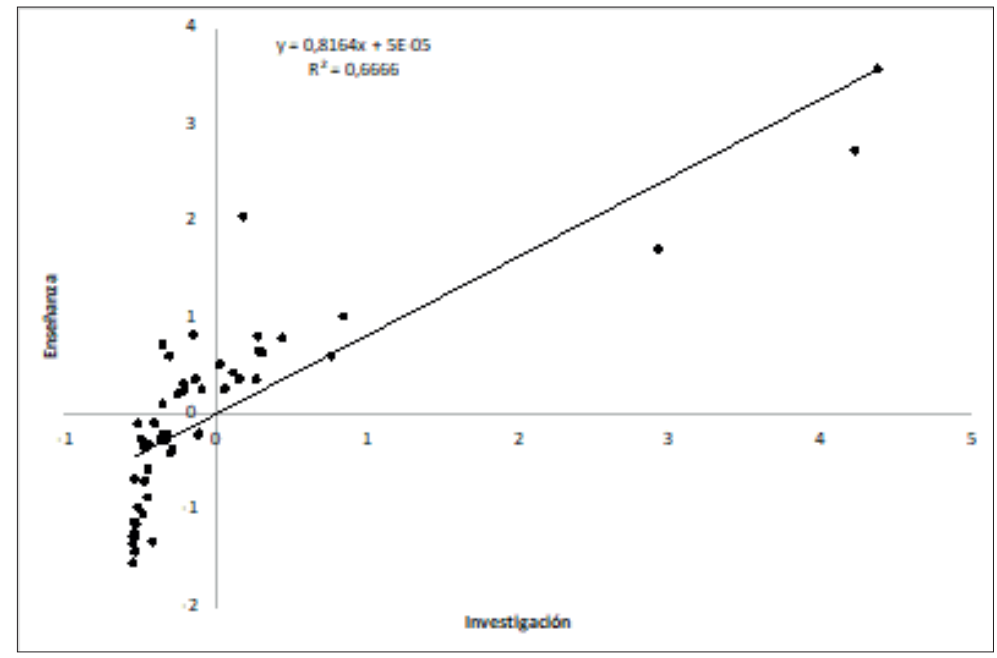

Figura 2. Distribución de variables latentes investigación y enseñanza de las universidades chilenas.

Fuente: Elaboración propia.

En este caso, se puede ver que existe un comportamiento muy heterogéneo entre las instituciones, no obstante, se identifican tres grupos: universidades que presentan valores positivos para ambas VL; universidades que presentan valores negativos para la VL investigación y valores positivos para la VL enseñanza; universidades con valores negativos para ambas VL. No se presentan universidades con valores positivos en investigación y negativos en enseñanza. 
En la Tabla 5 se resumen los grupos de universidades identificadas.

\section{Tabla 5}

Universidades según valores positivos y negativos de VL

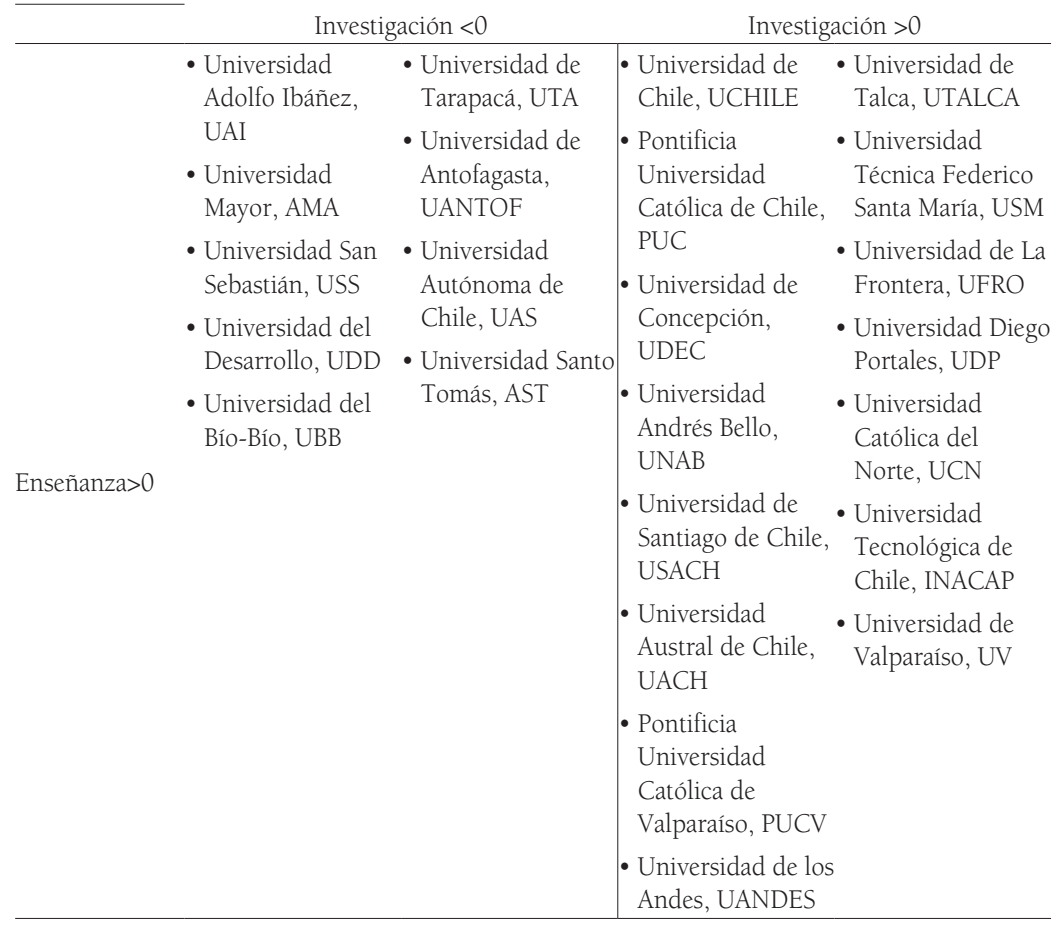



ANÁLISIS CON MODELOS DE ECUACIONES ESTRUCTURALES - C. Mondaca, J. Lopatinsky, A. Montecinos, J. Rojas-Mora

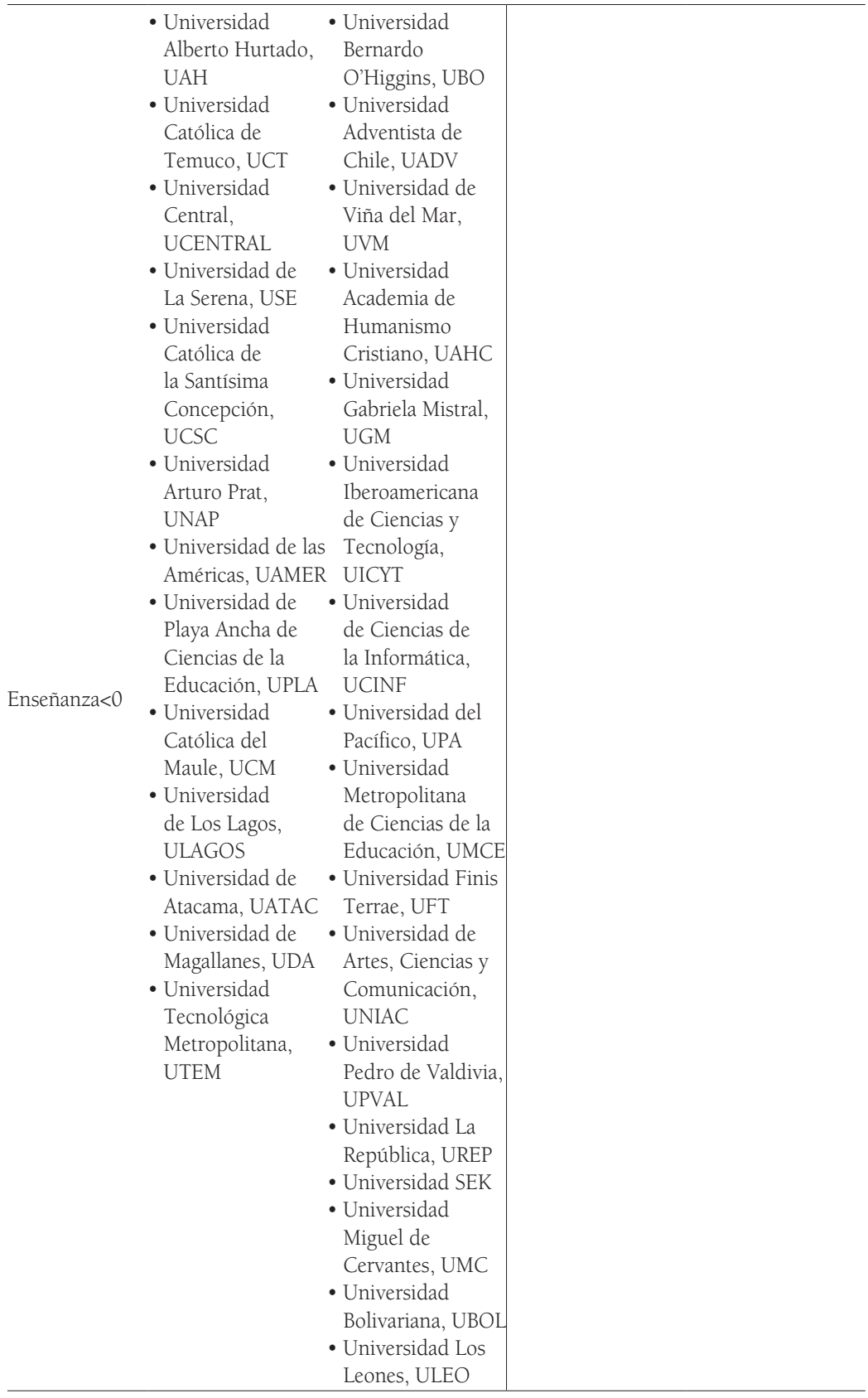

Fuente: Elaboración propia. 
De la tabla anterior, en el primer cuadrante se puede identificar a 15 universidades que presentan valores positivos para ambas variables latentes (investigación y enseñanza), y muestra un grupo reducido de universidades que desarrollan en forma amplia ambas misiones; aunque existe una distancia importante entre UCHILE y PUC respecto de las demás universidades.

En el segundo cuadrante — que agrupa las universidades con VL negativas en investigación y positivos en enseñanza—, se pueden identificar nueve universidades; ello evidencia que algunas de ellas han privilegiado desarrollar con mayor intensidad su misión de enseñanza, destacando las universidades: UAI, AMA y USS.

En el tercer cuadrante, se puede observar al grupo de universidades que presentan valores negativos en ambas VL. En este caso se identifican 30 universidades que, dado que presentan valores por debajo de la media en ambas VL, se pueden asociar a universidades que muestran un bajo nivel de desarrollo en ambas misiones (investigación y enseñanza). Estos casos exhiben bajos valores en la mayoría de los indicadores asociados al modelo, en particular, bajos niveles de productividad científica y escasa o nula transferencia e innovación; además de bajos niveles en acreditación y un número menor de docentes con posgrado; también presentan una baja dotación de recursos para la enseñanza, y un desarrollo principalmente orientado a la docencia de pregrado.

Se debe comentar que en el sistema chileno no existen universidades con un perfil exclusivamente investigador, como se puede encontrar en otros países, y que ocuparían el cuarto cuadrante.

En la Figura 3 se puede observar la correlación entre el grado de desarrollo de las universidades y sus misiones, en donde se puede apreciar que de las 22 universidades que muestran un valor positivo en su índice de desarrollo, solo 15 destacan por su actividad de investigación, y cinco de ellas lo hacen en forma amplia (UCHILE, PUC, UDEC, USACH, UACH); es decir, presentan producción científica y acciones de transferencia e innovación en forma destacada. 

ANÁLISIS CON MODELOS DE ECUACIONES ESTRUCTURALES - C. Mondaca, J. Lopatinsky,
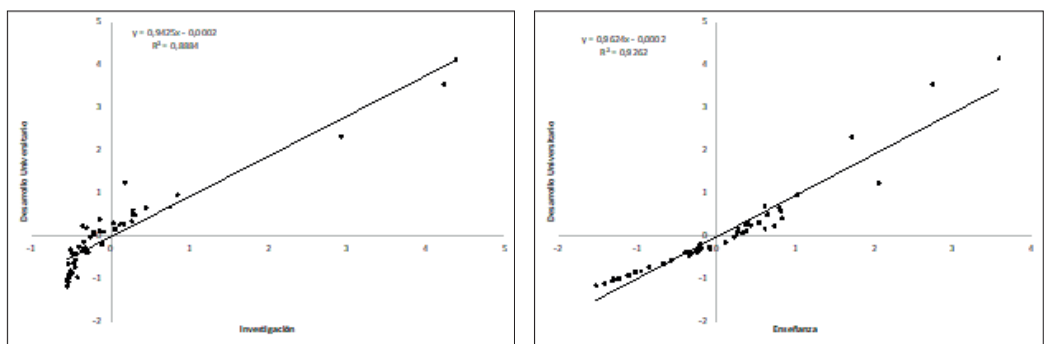

Figura 3. Correlación entre investigación-desarrollo y enseñanza-desarrollo. Fuente: Elaboración propia.

En el caso de la enseñanza y el grado de desarrollo, se puede apreciar mayor uniformidad, y a pesar de que existen cinco universidades que se alejan en forma significativa por su alto nivel de desarrollo y actividad de enseñanza (UCHILE, PUC, UNAB, UDEC, USACH), existen 24 universidades que desarrollan su segunda misión por sobre la media (valor positivo del constructo); es decir, presentan docencia de excelencia, un alto grado de recursos y espacios para la enseñanza, y un flujo de estudiantes en sus programas de pre y posgrado. El resto de universidades se diferencia por presentar menores niveles de acreditación y académicos con posgrado, y orientarse principalmente a la docencia de pregrado.

En la Figura 4 se pueden ver las variables latentes determinadas por el modelo estructural para cada universidad, y en la Tabla 7 del Anexo un ranking construido en función de ellas.

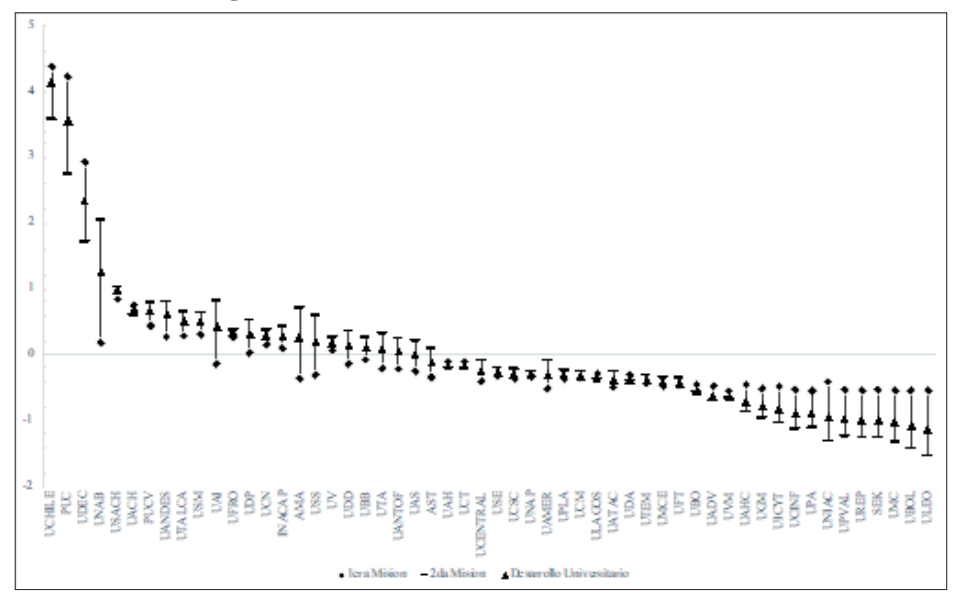

Figura 4. Índice desarrollo de las universidades chilenas (2017).

Fuente: Elaboración propia. 
En la Figura 4 se pueden identificar 10 universidades (que representan 18,5\% del total) que muestran valores positivos en su índice de desarrollo y en sus actividades de investigación y enseñanza, las cuales se mencionan a continuación: UCHILE, PUC, UDEC, UNAB, USACH, UACH, PUCV, UANDES, UTALCA, USM.

Las universidades mencionadas destacan por su alto nivel de producción científica; capacidad de transferir investigación aplicada e innovación; docencia de excelencia debido a su mayor cantidad de académicos con grado de doctor, alto nivel de acreditación y mayor cantidad de recursos físicos y financieros dedicados a la enseñanza; además de un flujo importante de estudiantes que se forma en sus programas de pre y posgrado. Se puede ver que este grupo es diverso, formado por universidades públicas y privadas, tradicionales y de reciente formación, localizadas en la Región Metropolitana de Santiago y en otras regiones del país.

Por otra parte, se aprecia que las discrepancias entre universidades se deben a las diferencias en su productividad científica e intensidad en el flujo de estudiantes (particularmente en posgrado), lo que define las distancias entre instituciones. Esta situación muestra un rasgo característico del sistema chileno de universidades, en donde las más aventajadas desarrollan en forma amplia sus misiones, y presentan una mayor productividad científica y mayor docencia, particularmente de posgrado.

También se puede observar en la Figura 4 que existen 12 universidades (que representan 22\% del total) que desarrollan en forma intensiva la enseñanza y en menor medida su actividad de investigación, focalizando sus esfuerzos en la docencia de pregrado.

Además, se puede identificar un grupo de 32 universidades (que representan el 59\% del total), las cuales muestran un bajo nivel de desarrollo; es decir, un valor negativo en el índice general. De este grupo, 30 presentan valores negativos en ambas misiones, es decir, tanto en la actividad de investigación como en las de enseñanza. Estas universidades se caracterizan por tener baja productividad científica, casi nula transferencia e innovación, un bajo nivel de acreditación, 
destinan una menor cantidad de recursos a la enseñanza y tienen una fuerte orientación hacia la docencia de pregrado.

Resumiendo, los resultados muestran que solo el 19\% del total de universidades consideradas en el estudio, desarrolla en forma amplia las actividades de investigación y enseñanza, destacando por su alto nivel de producción científica, capacidad de transferir conocimiento e innovación, y que desarrollan una docencia de excelencia. El 22\% de las universidades despliega en forma intensiva la enseñanza y en menor intensidad su actividad de investigación, focalizando sus esfuerzos en la docencia de pregrado. El 59\% de las universidades estudiadas muestra un bajo nivel de desarrollo en ambas misiones, las cuales presentan una brecha significativa en el ámbito de la enseñanza y en la investigación.

Finalmente se comparó el ranking construido con la VL desarrollo, frente a uno de los rankings más conocidos —el Ranking de calidad de las universidades chilenas-RCUCh $-{ }^{8}$. En este caso solo se pudo realizar la comparación para 45 universidades, que cuentan con una posición en el ranking y que están incluidas en este trabajo.

Los resultados muestran que al considerar las primeras 10 universidades, existe una coincidencia en un 70\% del grupo de universidades mejor posicionadas, aunque con órdenes levemente diferentes. Si consideramos a las 20 primeras universidades, la coincidencia en el tramo es de un $75 \%$. Sin embargo, se puede observar que existen universidades que presentan posiciones diametralmente opuestas en los rankings. Las universidades UNAB e INACAP presentan una notable mejor posición que la asignada por el ranking RCUCh; al mismo tiempo que la UBB, está mejor posicionada en el ranking utilizado para la comparación, que la posición determinada en este estudio. Las coincidencias en las posiciones con un margen de \pm una posición, es de un 26,6\%.

Las diferencias existentes entre el ranking construido en este trabajo y el ranking RCUCh muestran que las universidades más

Esto puede verse en detalle en la Tabla 7 del Anexo. 
desarrolladas —debido a que presentan un alto grado de progreso en todas las misiones y actividades—, generalmente estarán posicionadas en lugares destacados independientemente de la metodología, indicadores, y constructos considerados. No se puede decir lo mismo de las demás universidades, en donde dependiendo de los indicadores, dimensiones y enfoque de análisis, algunas de ellas pueden cambiar de posición en forma importante, viéndose unas beneficiadas y otras perjudicadas.

Los resultados de este trabajo pueden ser de utilidad en el ámbito de las políticas públicas vinculadas a mejorar el sistema de educación universitaria. En este contexto, se recomiendan acciones que ayuden a disminuir la brecha existente entre las universidades que lideran el sistema chileno (UCHILE y PUC), respecto de las demás que desarrollan sus misiones en forma amplia; y revertir el efecto centrípeto de la Región Metropolitana respecto de las universidades de excelencia que se localizan en otras regiones del país. Esto se puede lograr con la provisión de recursos exclusivos para el desarrollo regional del sistema universitario chileno, vía financiamiento para la investigación con fondos regionales de concurso exclusivo para ellas, dada la menor capacidad que tienen de competir por fondos públicos respecto de las universidades líderes del sistema; además de fondos adicionales para fortalecer la captación y el mantenimiento del capital humano avanzado en regiones, facilitando la convergencia en el mediano plazo de las universidades regionales hacia las universidades líderes.

También son necesarias acciones para disminuir la brecha existente entre las universidades que desarrollan en forma amplia sus misiones de aquellas que presentan un bajo nivel de desarrollo, y que en el caso de Chile representan la mayoría de las universidades analizadas. En este sentido, es urgente aumentar los estándares de calidad del sistema universitario, con normativas que se enfoquen en la dotación y uso de los recursos públicos, que fijen estándares de calidad y excelencia en la infraestructura y personal vinculado con la enseñanza, de modo de disminuir las amplias diferencias actualmente existentes entre universidades. 
También se considera importante condicionar el financiamiento a que las universidades realicen actividad de investigación y de transferencia e innovación como parte de su quehacer institucional y, de este modo, transitar hacia un desarrollo integral de las universidades chilenas.

\section{Conclusiones}

En este trabajo se ha modelado el grado de desarrollo que presentan las universidades usando ecuaciones estructurales resueltas con mínimos cuadrados parciales PLS-SEM, utilizando datos del año 2017 para 54 universidades de Chile. Este indicador mide el grado de desarrollo valorando de forma cuantitativa las misiones (investigación y enseñanza) realizadas por la universidad, y cómo estas contribuyen a su posición final.

Los resultados muestran que un porcentaje mayoritario de universidades en Chile no desarrolla en forma amplia las misiones que tradicionalmente la sociedad espera de ellas, y expresan una intensiva orientación hacia las actividades de enseñanza, preferentemente de docencia de pregrado. Esto probablemente es consecuencia de la orientación del sistema chileno a desarrollar la educación universitaria como un bien de consumo, y en donde los incentivos monetarios se despliegan preferentemente para el desarrollo de la misión de enseñanza, dada la actual estructura de financiamiento del sistema de educación universitario, lo que debería cambiar si se desea aumentar los niveles de productividad científica y transferencia e innovación del país.

En este contexto, el sistema de educación universitaria chileno requiere de medidas que permitan disminuir la brecha existente entre universidades, particularmente entre las que lideran el sistema chileno y las que presentan un bajo nivel de desarrollo, destinando recursos para que las universidades mejoren su productividad científica y aumenten sus acciones de transferencia e innovación como partes de su quehacer, y así transitar hacia un desarrollo integral.

Como propuesta de trabajos futuros, se puede ampliar el modelo para que incorpore las actividades de extensión, debido al impacto que estas pueden tener en el entorno inmediato de las universidades 
y su capacidad de transferir su quehacer. La dimensión de extensión se enfoca en evaluar aspectos tales como: la vinculación con empresas para prestación de servicios y prácticas profesionales; convenios de colaboración e intercambio académico; vinculación con exalumnos para actividades de educación continua y perfeccionamiento, entre otros aspectos que pueden fortalecer la transferencia e investigación aplicada en las universidades (Hernández et al., 2014).

También se puede mejorar el modelo para incluir el grado de eficiencia con que las universidades realizan sus actividades de docencia, incluyendo variables como la tasa de titulación de los alumnos, indicador que tiene relación con la eficiencia del sistema o la relación remuneración académicos/ingresos operacionales, que podría entregar datos acerca de la importancia de los académicos en la institución. También se puede incluir la calidad de los input en el modelo, como por ejemplo, la excelencia académica de los alumnos que ingresan, utilizando indicadores como el ranking de los alumnos en su colegio o sus puntajes PSU, entre otras variables a considerar.

\section{Referencias}

Becker, J. M., Klein, K., \& Wetzels, M. (2012). Hierarchical latent variable models in PLS-SEM: Guidelines for using reflective-formative type models. Long Range Planning, 45(5-6), 359-394. https://doi. org/10.1016/j.lrp.2012.10.001

Del Barrio-García, S. y Luque-Martínez, T. (2012). Análisis de ecuaciones estructurales. En T. Luque-Martínez (Ed.), Técnicas de análisis de datos de investigación de mercado (pp. 489-577). Madrid: Ed. Pirámide.

Etzkowitz, H. \& Leydesdorff, L. (2000). The dynamics of innovation: From National Systems and "Mode 2" to a Triple Helix of university-industrygovernment relations. Research Policy, 29(2), 109-123. https://doi. org/10.1016/s0048-7333(99)00055-4

Fornell, C. \& Larcker, D. F. (1981). Evaluating structural equation models with unobservable variables and measurement error. Journal of Marketing Research, 18(1), 39-50. http://dx.doi.org/10.2307/3151312

Gasset, J. O. (1946). Mission of the university. London: Routledge.

Hair, J. F., Hult, G. T., Ringle, C. M., \& Sarstedt, M. (2017). A primer on partial least squares structural equation modelling (PLS-SEM) $(2 \mathrm{ed}$.). London: Sage Publication. 
312 MEDICIÓN DEL NIVEL DE DESARROLLO DE LAS UNIVERSIDADES CHILENAS: UN ANÁLISIS CON MODELOS DE ECUACIONES ESTRUCTURALES - C. Mondaca, J. Lopatinsky, A. Montecinos, J. Rojas-Mora

Hazelkorn, E. (2011). Rankings and the reshaping of higher education. New York: Palgrave Macmillan.

Hernández, P. M., Leyva, S. L., Márquez, C. Z., y Cerda, A. B. (2014). Evaluación de la calidad de la educación superior en México: comparación de los indicadores de rankings universitarios nacionales e internacionales. RIESED-Revista Internacional de Estudios sobre Sistemas Educativos, 2(4), 35-51. Recuperado de http://www.riesed.org/index. php/RIESED/article/view/63

Jajo, N. K. \& Harrison, J. (2014). World university ranking systems: An alternative approach using partial least squares path modelling. Journal of Higher Education Policy and Management, 36(5), 471-482. https://doi. org/10.1080/1360080x.2014.936090

Luque-Martínez, T. \& del Barrio-García, S. (2016). Constructing a synthetic indicator of research activity. Scientometrics, 108(3), 1049-1064. https://doi.org/10.1007/s11192-016-2037-8

Luque-Martínez, T. \& Doña-Toledo, L. (2013). What do graduates think? An analysis of intention to repeat the same studies and university. Journal of Marketing for High Education, 23(1), 62-89. https://doi.org/10.1080/ 08841241.2013 .812589

Martínez Rizo, F. (2011). Los rankings de universidades: una visión crítica. Revista de la Educación Superior, 40(157), 77-97. Recuperado de https://www.redalyc.org/pdf/604/60420223004.pdf

Martínez, F., Robles, H., Hernández, J. M., Zendejas, L., y Pérez, M. (2007). Propuesta y experiencias para desarrollar un sistema nacional de indicadores educativos. México DF: Instituto Nacional para la Evaluación de la Educación. Recuperado de https://www.inee.edu.mx/wp-content/ uploads/2018/12/P1C223.pdf

Muñoz, M. y Blanco, C. (2013). Una taxonomía de las universidades chilenas. Calidad en la Educación, 38, 181-213. https://doi.org/10.4067/s071845652013000100005

Pacheco, M. (1997). Misión de las universidades. Revista Chilena de Derecho, 24(1), 49-68. Recuperado de https://dialnet.unirioja.es/descarga/ articulo/2650016.pdf

Pérez Esparrells, C. y Gómez Sancho, J. (2010). Los rankings internacionales de las instituciones de educación superior y las clasificaciones universitarias en España: visión panorámica y prospectiva de futuro. Recuperado de https://www.researchgate.net/publication/277270044_Los_rankings_ internacionales_de_las_instituciones_de_educacion_superior_y_las_ clasificaciones_universitarias_en_Espana_vision_panoramica_y_ prospectiva_de_futuro 
Quiles, O. y de Gracia, E. (2015). Calidad y evaluación de la educación superior. Una perspectiva transnacional a través de los rankings. DEDiCA. Revista de Educación e Humanidades, 8, 155-174. Recuperado de http://digibug.ugr.es/handle/10481/37459

Reyes, C. (2016). Medición de la calidad universitaria en Chile: la influencia de los rankings. Calidad en la Educación, 44, 158-196. https://doi. org/10.4067/s0718-45652016000100007

Ringle, C. M., Wende, S., \& Becker, J. M. (2015). SmartPLS 3 [Boenningstedt: SmartPLS GmbH]. Recuperado de http://www.smartpls.com

Sánchez-Barrioluengo, M. (2014). Articulating the 'three-missions' in Spanish universities. Research Policy, 43(10), 1760-1773. https://doi. org/10.1016/j.respol.2014.06.001

Sanz-Casado, E., García-Zorita, C., Serrano-López, A., Efraín-García, E., y De Filippo, D. (2013). Rankings nacionales elaborados a partir de múltiples indicadores frente a los índices sintéticos. Revista Española de Documentación Científica, 36(3), 1-18. https://doi.org/10.3989/ redc.2013.3.1.023

Sarstedt, M., Ringle, C. M. \& Hair, J. F. (2014). PLS-SEM: Looking back and moving forward. Long Range Planning, 47(3), 132-137. https://doi. org/10.1016/j.lrp.2014.02.008

Scott, J. C. (2006). The mission of the university: Medieval to postmodern transformations, The Journal of Higher Education, 77(1), 1-39. https://doi.org/10.1080/00221546.2006.11778917

Taylor, P. \& Braddock, R. (2007). International university ranking systems and the idea of university excellence. Journal of Higher Education Policy and Management, 29(3), 245-260. https://doi. org/10.1080/13600800701457855

Usher, A. y Savino, M. (2018). Estudio global de los rankings universitarios. Calidad en la Educación, 25, 33-53. https://doi.org/10.31619/caledu. n25.252

Vinzi, V. E., Chin, W. W., Henseler, J., \& Wang, H. (2010). Handbook of partial least squares: Concepts, methods and applications. Berlin: Springer.

Recibido: 12/03/2019

Aceptado:13/06/2019 

ANÁLISIS CON MODELOS DE ECUACIONES ESTRUCTURALES - C. Mondaca, J. Lopatinsky, A. Montecinos, J. Rojas-Mora

\section{ANEXO}

Tabla 6

Definición de las variables usadas en el modelo y estadística descriptiva

\begin{tabular}{|c|c|c|c|c|c|c|c|}
\hline Constructo & Indicador & Descripción & Media & Mediana & Mín. & Máx. & D.S. \\
\hline \multirow[t]{3}{*}{$\begin{array}{l}\text { Producción } \\
\text { científica }\end{array}$} & $\begin{array}{l}\mathrm{N}^{o} \text { proyectos } \\
\text { Fondecyt }\end{array}$ & $\begin{array}{l}\text { Número de } \\
\text { proyectos } \\
\text { Fondecyt } \\
\text { (últimos } 5 \\
\text { años) }\end{array}$ & 9,4 & 2 & 0 & 106 & 20,1 \\
\hline & $\begin{array}{l}\mathrm{N}^{\mathrm{o}} \text { de artículos } \\
\text { WoS }\end{array}$ & $\begin{array}{l}\text { Número de } \\
\text { artículos WoS } \\
\text { año } 2017\end{array}$ & 248,2 & 95 & 0 & 2.305 & 443,5 \\
\hline & $\begin{array}{l}\mathrm{N}^{\circ} \text { de artículos } \\
\text { SciELO no ISI }\end{array}$ & $\begin{array}{l}\text { Número de } \\
\text { artículos } \\
\text { SciELO no ISI } \\
\text { año } 2017\end{array}$ & 30,5 & 16 & 0 & 279 & 481,5 \\
\hline \multirow[t]{3}{*}{$\begin{array}{l}\text { Transferencia } \\
\text { e innovación }\end{array}$} & $\begin{array}{l}N^{o} \text { total de } \\
\text { patentes }\end{array}$ & $\begin{array}{l}\text { Número total } \\
\text { de patentes }\end{array}$ & 7,4 & 0 & 0 & 168 & 25,6 \\
\hline & $\begin{array}{l}\mathrm{N}^{\circ} \text { de proyectos } \\
\text { Fondef }\end{array}$ & $\begin{array}{l}\text { Número de } \\
\text { proyectos } \\
\text { Fondef } \\
\text { (últimos } 5 \\
\text { años) }\end{array}$ & 13,6 & 2 & 0 & 131 & 28,6 \\
\hline & $\begin{array}{l}\text { Ingresos por } \\
\text { prestaciones de } \\
\text { servicios }\end{array}$ & $\begin{array}{l}\text { Porcentaje de } \\
\text { los ingresos } \\
\text { totales que } \\
\text { corresponden } \\
\text { a prestación } \\
\text { de servicios }\end{array}$ & 0,058 & 0,016 & 0 & 0,558 & 0,117 \\
\hline \multirow[t]{4}{*}{$\begin{array}{l}\text { Docencia de } \\
\text { excelencia }\end{array}$} & $\mathrm{N}^{\mathrm{o}}$ doctores JC & $\begin{array}{l}\text { Número de } \\
\text { docentes } \\
\text { con grado } \\
\text { de doctor } \\
\text { a jornada } \\
\text { completa }\end{array}$ & 208,5 & 144 & 0 & 1.346 & 268,1 \\
\hline & $\mathrm{N}^{\circ}$ magíster JC & $\begin{array}{l}\text { Número de } \\
\text { docentes } \\
\text { con grado } \\
\text { de magíster } \\
\text { a jornada } \\
\text { completa }\end{array}$ & 428,8 & 306 & 25 & 1984 & 376,4 \\
\hline & $\begin{array}{l}\text { Años de } \\
\text { acreditación }\end{array}$ & $\begin{array}{l}\text { Años de } \\
\text { acreditación } \\
\text { de la } \\
\text { universidad }\end{array}$ & 3,7 & 4 & 0 & 7 & 2,02 \\
\hline & Áreas acreditadas & $\begin{array}{l}\text { Número } \\
\text { de áreas } \\
\text { acreditadas } \\
\text { por la } \\
\text { universidad }\end{array}$ & 3,0 & 3 & 0 & 5 & 1,69 \\
\hline
\end{tabular}




\begin{tabular}{|c|c|c|c|c|c|c|c|}
\hline \multirow[t]{3}{*}{$\begin{array}{l}\text { Recursos y } \\
\text { espacios para } \\
\text { la enseñanza }\end{array}$} & $\mathrm{M}^{2}$ de inmueble & $\begin{array}{l}\text { Metros } \\
\text { cuadrados de } \\
\text { inmueble por } \\
\text { alumno }\end{array}$ & 7,4 & 6,9 & 0,97 & 17,24 & 3,31 \\
\hline & $\begin{array}{l}\text { Ingresos } \\
\text { operacionales }\end{array}$ & $\begin{array}{l}\text { Ingresos } \\
\text { operacionales } \\
\text { por alumno }\end{array}$ & $7.765,7$ & $6.883,8$ & 868,3 & $33.676,1$ & $5.698,7$ \\
\hline & $\begin{array}{l}\text { Remuneraciones } \\
\text { de académicos }\end{array}$ & $\begin{array}{l}\text { Remuneración } \\
\text { mensual de } \\
\text { académicos }\end{array}$ & $1.181,9$ & 989,0 & 218,6 & $3.137,6$ & 749,0 \\
\hline \multirow[t]{4}{*}{$\begin{array}{l}\text { Procesos } \\
\text { formativos }\end{array}$} & $\begin{array}{l}\text { Matricula } \\
\text { pregrado }\end{array}$ & $\begin{array}{l}\text { Número de } \\
\text { estudiantes } \\
\text { matriculados } \\
\text { en pregrado } \\
\text { en } 2017\end{array}$ & $12.030,8$ & $8.757,0$ & 546 & 43.315 & $9.352,9$ \\
\hline & $\begin{array}{l}\text { Matricula } \\
\text { posgrado }\end{array}$ & $\begin{array}{l}\text { Número de } \\
\text { estudiantes } \\
\text { matriculados } \\
\text { en posgrado } \\
\text { en } 2017 \\
\end{array}$ & 896,7 & 463 & 0 & 6.801 & $1.253,4$ \\
\hline & $\begin{array}{l}\text { Titulados } \\
\text { pregrado }\end{array}$ & $\begin{array}{l}\text { Número de } \\
\text { estudiantes } \\
\text { titulados en } \\
\text { pregrado }\end{array}$ & $1.663,9$ & $1.118,0$ & 124 & 6.333 & $1.349,0$ \\
\hline & $\begin{array}{l}\text { Graduados } \\
\text { posgrado }\end{array}$ & $\begin{array}{l}\text { Número de } \\
\text { alumnos } \\
\text { graduados en } \\
\text { posgrado }\end{array}$ & 268,3 & 96 & 0 & 1.915 & 404,9 \\
\hline
\end{tabular}

Fuente: Elaboración propia. 
316 MEDICIÓN DEL NIVEL DE DESARROLLO DE LAS UNIVERSIDADES CHILENAS: UN ANÁLISIS CON MODELOS DE ECUACIONES ESTRUCTURALES - C. Mondaca, J. Lopatinsky, A. Montecinos, J. Rojas-Mora

Tabla 7

Ranking de universidades según complejidad, dimensión investigadora y dimensión docente de la universidad

\begin{tabular}{|c|c|c|c|c|c|c|c|c|}
\hline \multirow[t]{2}{*}{ 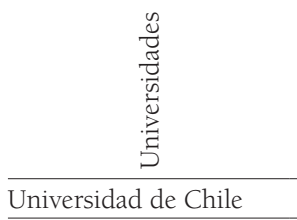 } & \multirow{2}{*}{$\frac{\overbrace{\infty}^{\frac{\pi}{\infty}}}{\text { UCHILE }}$} & \multirow{2}{*}{ 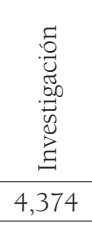 } & \multirow{2}{*}{ 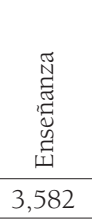 } & \multirow{2}{*}{ 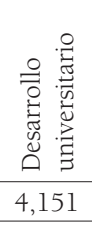 } & \multicolumn{3}{|c|}{ 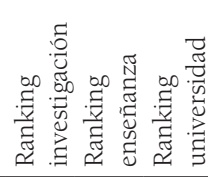 } & \multirow{2}{*}{ 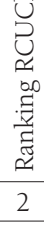 } \\
\hline & & & & & 1 & 1 & 1 & \\
\hline $\begin{array}{l}\text { Pontificia Universidad } \\
\text { Católica de Chile }\end{array}$ & PUC & 4,221 & 2,737 & 3,567 & 2 & 2 & 2 & 1 \\
\hline $\begin{array}{l}\text { Universidad de } \\
\text { Concepción }\end{array}$ & UDEC & 2,917 & 1,712 & 2,333 & 3 & 4 & 3 & 3 \\
\hline $\begin{array}{l}\text { Universidad Andrés } \\
\text { Bello }\end{array}$ & UNAB & 0,176 & 2,052 & 1,262 & 11 & 3 & 4 & 31 \\
\hline $\begin{array}{l}\text { Universidad de Santiago } \\
\text { de Chile }\end{array}$ & USACH & 0,841 & 1,019 & 0,975 & 4 & 5 & 5 & 14 \\
\hline $\begin{array}{l}\text { Universidad Austral de } \\
\text { Chile }\end{array}$ & $\mathrm{UACH}$ & 0,756 & 0,603 & 0,701 & 5 & 13 & 6 & 5 \\
\hline $\begin{array}{l}\text { Pontificia Universidad } \\
\text { Católica de Valparaíso }\end{array}$ & PUCV & 0,440 & 0,785 & 0,666 & 6 & 8 & 7 & 7 \\
\hline $\begin{array}{l}\text { Universidad de los } \\
\text { Andes }\end{array}$ & UANDES & 0,274 & 0,810 & 0,614 & 9 & 7 & 8 & 13 \\
\hline Universidad de Talca & UTALCA & 0,282 & 0,649 & 0,516 & 8 & 10 & 9 & 6 \\
\hline $\begin{array}{l}\text { Universidad Técnica } \\
\text { Federico Santa María }\end{array}$ & USM & 0,300 & 0,633 & 0,504 & 7 & 11 & 10 & 10 \\
\hline $\begin{array}{l}\text { Universidad Adolfo } \\
\text { Ibáñez }\end{array}$ & UAI & $-0,148$ & 0,824 & 0,423 & 20 & 6 & 11 & 15 \\
\hline $\begin{array}{l}\text { Universidad de la } \\
\text { Frontera }\end{array}$ & UFRO & 0,265 & 0,374 & 0,346 & 10 & 16 & 12 & 8 \\
\hline $\begin{array}{l}\text { Universidad Diego } \\
\text { Portales }\end{array}$ & UDP & 0,028 & 0,528 & 0,32 & 15 & 14 & 13 & 21 \\
\hline $\begin{array}{l}\text { Universidad Católica del } \\
\text { Norte }\end{array}$ & UCN & 0,156 & 0,373 & 0,293 & 12 & 17 & 14 & 14 \\
\hline $\begin{array}{l}\text { Universidad Tecnológica } \\
\text { de Chile }\end{array}$ & INACAP & 0,107 & 0,431 & 0,277 & 13 & 15 & 15 & 32 \\
\hline Universidad Mayor & AMA & $-0,358$ & 0,720 & 0,254 & 30 & 9 & 16 & 28 \\
\hline $\begin{array}{l}\text { Universidad San } \\
\text { Sebastián }\end{array}$ & USS & $-0,309$ & 0,606 & 0,195 & 26 & 12 & 17 & 27 \\
\hline $\begin{array}{l}\text { Universidad de } \\
\text { Valparaíso }\end{array}$ & UV & 0,052 & 0,273 & 0,182 & 14 & 20 & 18 & 17 \\
\hline $\begin{array}{l}\text { Universidad del } \\
\text { Desarrollo }\end{array}$ & UDD & $-0,143$ & 0,363 & 0,138 & 19 & 18 & 19 & 11 \\
\hline Universidad del Bío-bío & UBB & $-0,095$ & 0,263 & 0,109 & 16 & 21 & 20 & 4 \\
\hline Universidad de Tarapacá & UTA & $-0,214$ & 0,323 & 0,095 & 21 & 19 & 21 & 9 \\
\hline $\begin{array}{l}\text { Universidad de } \\
\text { Antofagasta }\end{array}$ & UANTOF & $-0,216$ & 0,246 & 0,050 & 22 & 22 & 22 & 18 \\
\hline $\begin{array}{l}\text { Universidad Autónoma } \\
\text { de Chile }\end{array}$ & UAS & $-0,263$ & 0,218 & $-0,011$ & 23 & 23 & 23 & 23 \\
\hline
\end{tabular}




\begin{tabular}{|c|c|c|c|c|c|c|c|c|}
\hline $\begin{array}{l}\text { Universidad Santo } \\
\text { Tomás }\end{array}$ & AST & $-0,351$ & 0,105 & $-0,121$ & 29 & 24 & 24 & 42 \\
\hline $\begin{array}{l}\text { Universidad Alberto } \\
\text { Hurtado }\end{array}$ & $\mathrm{UAH}$ & $-0,116$ & $-0,201$ & $-0,173$ & 18 & 27 & 25 & 24 \\
\hline $\begin{array}{l}\text { Universidad Católica de } \\
\text { Temuco }\end{array}$ & UCT & $-0,115$ & $-0,210$ & $-0,181$ & 17 & 29 & 26 & 30 \\
\hline $\begin{array}{l}\text { Universidad Central de } \\
\text { Chile }\end{array}$ & UCENTRAL & $-0,412$ & $-0,092$ & $-0,249$ & 34 & 26 & 27 & 35 \\
\hline $\begin{array}{l}\text { Universidad de La } \\
\text { Serena }\end{array}$ & USE & $-0,323$ & $-0,207$ & $-0,267$ & 27 & 28 & 28 & 25 \\
\hline $\begin{array}{l}\text { Universidad Católica de } \\
\text { la Santísima Concepción }\end{array}$ & UCSC & $-0,359$ & $-0,215$ & $-0,298$ & 31 & 30 & 29 & 33 \\
\hline Universidad Arturo Prat & UNAP & $-0,330$ & $-0,254$ & $-0,302$ & 28 & 32 & 30 & 43 \\
\hline $\begin{array}{l}\text { Universidad de las } \\
\text { Américas }\end{array}$ & UAMER & $-0,514$ & $-0,084$ & $-0,310$ & 44 & 25 & 31 & 44 \\
\hline $\begin{array}{l}\text { Universidad de Playa } \\
\text { Ancha de Ciencias de la } \\
\text { Educación }\end{array}$ & UPLA & $-0,364$ & $-0,243$ & $-0,311$ & 33 & 31 & 32 & 20 \\
\hline $\begin{array}{l}\text { Universidad Católica del } \\
\text { Maule }\end{array}$ & UCM & $-0,359$ & $-0,262$ & $-0,318$ & 32 & 34 & 33 & 19 \\
\hline $\begin{array}{l}\text { Universidad de Los } \\
\text { Lagos }\end{array}$ & ULAGOS & $-0,296$ & $-0,369$ & $-0,367$ & 24 & 38 & 34 & 41 \\
\hline Universidad de Atacama & UATAC & $-0,492$ & $-0,256$ & $-0,371$ & 43 & 33 & 35 & 37 \\
\hline $\begin{array}{l}\text { Universidad de } \\
\text { Magallanes }\end{array}$ & UDA & $-0,305$ & $-0,404$ & $-0,375$ & 25 & 39 & 36 & 34 \\
\hline $\begin{array}{l}\text { Universidad Tecnológica } \\
\text { Metropolitana }\end{array}$ & UTEM & $-0,444$ & $-0,320$ & $-0,392$ & 36 & 35 & 37 & 38 \\
\hline $\begin{array}{l}\text { Universidad } \\
\text { Metropolitana de } \\
\text { Ciencias de la Educación }\end{array}$ & UMCE & $-0,475$ & $-0,354$ & $-0,418$ & 39 & 37 & 38 & 16 \\
\hline Universidad Finis Terrae & UFT & $-0,480$ & $-0,347$ & $-0,424$ & 41 & 36 & 39 & 36 \\
\hline $\begin{array}{l}\text { Universidad Bernardo } \\
\text { O'Higgins }\end{array}$ & UBO & $-0,454$ & $-0,571$ & $-0,540$ & 37 & 40 & 40 & 26 \\
\hline $\begin{array}{l}\text { Universidad Adventista } \\
\text { de Chile }\end{array}$ & UADV & $-0,479$ & $-0,694$ & $-0,623$ & 40 & 42 & 41 & 22 \\
\hline $\begin{array}{l}\text { Universidad de Viña } \\
\text { del Mar }\end{array}$ & UVM & $-0,544$ & $-0,668$ & $-0,646$ & 49 & 41 & 42 & 39 \\
\hline $\begin{array}{l}\text { Universidad Academia } \\
\text { de Humanismo Cristiano }\end{array}$ & UAHC & $-0,455$ & $-0,864$ & $-0,713$ & 38 & 43 & 43 & 40 \\
\hline $\begin{array}{l}\text { Universidad Gabriela } \\
\text { Mistral }\end{array}$ & UGM & $-0,514$ & $-0,956$ & $-0,793$ & 45 & 44 & 44 & 46 \\
\hline $\begin{array}{l}\text { Universidad } \\
\text { Iberoamericana de } \\
\text { Ciencias y Tecnología }\end{array}$ & UICYT & $-0,488$ & $-1,033$ & $-0,827$ & 42 & 45 & 45 & 45 \\
\hline $\begin{array}{l}\text { Universidad de Ciencias } \\
\text { de la Informática }\end{array}$ & UCINF & $-0,526$ & $-1,131$ & $-0,893$ & 46 & 47 & 46 & - \\
\hline Universidad del Pacífico & UPA & $-0,546$ & $-1,113$ & $-0,895$ & 50 & 46 & 47 & - \\
\hline $\begin{array}{l}\text { Universidad de } \\
\text { Artes, Ciencias y } \\
\text { Comunicación }\end{array}$ & UNIAC & $-0,423$ & $-1,321$ & $-0,962$ & 35 & 51 & 48 & - \\
\hline
\end{tabular}


318 MEDICIÓN DEL NIVEL DE DESARROLLO DE LAS UNIVERSIDADES CHILENAS: UN ANÁLISIS CON MODELOS DE ECUACIONES ESTRUCTURALES - C. Mondaca, J. Lopatinsky, A. Montecinos, J. Rojas-Mora

\begin{tabular}{lcccccccc}
\hline $\begin{array}{l}\text { Universidad Pedro de } \\
\text { Valdivia }\end{array}$ & UPVAL & $-0,543$ & $-1,239$ & $-0,973$ & 47 & 48 & 49 & - \\
\hline $\begin{array}{l}\text { Universidad La } \\
\text { República }\end{array}$ & UREP & $-0,550$ & $-1,264$ & $-0,989$ & 52 & 49 & 50 & - \\
\hline Universidad SEK & SEK & $-0,543$ & $-1,267$ & $-0,989$ & 48 & 50 & 51 & - \\
\hline $\begin{array}{l}\text { Universidad Miguel de } \\
\text { Cervantes }\end{array}$ & UMC & $-0,550$ & $-1,328$ & $-1,022$ & 53 & 52 & 52 & - \\
\hline Universidad Bolivariana & UBOL & $-0,546$ & $-1,425$ & $-1,082$ & 51 & 53 & 53 & - \\
\hline Universidad Los Leones & ULEO & $-0,550$ & $-1,537$ & $-1,148$ & 54 & 54 & 54 & - \\
\hline
\end{tabular}

Fuente: Elaboración propia. 